\title{
LMI-Based Neurocontroller for Guaranteed Cost Control of Uncertain Time-Delay Systems
}

\author{
Hiroaki Mukaidani \\ Graduate School of Education \\ Hiroshima University \\ 1-1-1 Kagamiyama, Higashi-Hiroshima \\ 739-8524 Japan. \\ Email: mukaida@hiroshima-u.ac.jp
}

\author{
Seishiro Sakaguchi and Toshio Tsuji \\ Graduate School of Engineering \\ Hiroshima University \\ 1-4-1 Kagamiyama, Higashi-Hiroshima \\ 739-8527 Japan. \\ Email: \{sakaguchi, tsuji\}@bsys.hiroshima-u.ac.jp
}

\begin{abstract}
This paper considers the application of the neural networks for the guaranteed cost control problem of discretetime uncertain systems that have in both state and input delays. In order to guarantee the stability of the closed-loop delay systems with the neurocontroller, the Linear Matrix Inequality (LMI) condition is established, while the neurocontroller is used to reduce the cost of the uncertain time-delay systems caused by the LMI design parameter. The novel contribution is that the neurocontroller is substituted for the additive gain perturbations. It is newly shown that although the neurocontroller is included in the uncertain discrete-time delay systems, the robust stability for the closed-loop systems and the reduction of the cost are attained. Finally, a numerical example is given to verify the efficiency.
\end{abstract}

\section{INTRODUCTION}

Recent advance in theory of Linear Matrix Inequality (LMI) has allowed a revisiting of the guaranteed cost control approach for the uncertain discrete-time delay systems [2], [3]. The LMI design method is a very well-known and powerful tool, it can not only efficiently find feasible and global solutions, but also easily handle various kinds of additional linear constraints. However, in [2], [3], the control gain variations [4] have not been considered. It is very important to study the robustness of the discrete-time delay systems with the control gain variations because any useful design procedure to generate a controller which also has sufficient room for readjustment of its coefficients is needed [4].

Up to now, neural networks (NNs) have been extensively studied to construct the intelligent control systems. Some control methodologies have been proposed by combining NNs with modern control theories [5], [6]. However, there is a possibility that $\mathrm{NN}$ can not stabilize systems because the general stability of the systems including NNs has not been considered [6]. Very recently, in order to ensure the robust stability of the neurocontroller, the additive gain perturbation approach has been used [8], [7]. As a result, it is shown that the neurocontroller succeeds in achieving the cost reduction and guaranteeing the robust stability for the systems. However, the guaranteed cost control for the uncertain discrete-time delay systems with the neurocontroller has not been investigated.

This paper investigates the guaranteed cost control for the discrete-time systems that have in both state and input timedelays. Firstly, a class of the fixed state feedback controller of the uncertain time-delay systems with the gain perturbations is newly derived and some sufficient conditions to design the guaranteed cost controller are established by means of LMI. In order to reduce the cost performance caused by the parameter uncertainties in the LMI, NNs are substituted for the additive gain perturbations. It should be noted that the NNs use not only the present state value but also the past state value of the uncertain systems. For this reason, the proposed method is suitable for uncertain discrete-time delay systems for reducing the cost. Finally, in order to verify the effectiveness of the proposed method, numerical examples are shown.

\section{PRELIMINARY}

Consider uncertain discrete-time systems that have in both the state and the input delays as follows

$$
\begin{aligned}
x(k+1)= & A(k) x(k)+A_{1}(k) x\left(k-d_{1}\right) \\
& +B_{1}(k) u(k)+B_{2}(k) u\left(k-d_{2}\right), \\
u(k)= & K(k) x(k),
\end{aligned}
$$

where $x(k) \in \Re^{m}$ is the state, $u(k) \in \Re^{n}$ is the control input, $d_{1}$ and $d_{2}$ are unknown constant integers representing the number of delay units in the state and input, respectively. Without loss of generality, we can assume that $0 \leq d_{i} \leq d_{i}{ }^{*}, i=1,2$, where $d_{i}{ }^{*}$ is known previously. $A(k), A_{1}(k), B_{i}(k), i=1,2$ can be described as follows

$$
\begin{aligned}
A(k) & =A+\Delta A(k), \quad A_{1}(k)=A_{1}+\Delta A_{1}(k), \\
B_{i}(k) & =B_{i}+\Delta B_{i}(k),
\end{aligned}
$$

where $A, A_{1}, B_{1}$ and $B_{2}$ are known real constant matrices of appropriate dimensions, $\Delta A(k), \Delta A_{1}(k), \Delta B_{1}(k)$ and $\Delta B_{2}(k)$ are uncertain matrices representing time-varying parameter uncertainties in the systems. These parameter uncertainties are assumed to be of the following form

$$
\begin{aligned}
& {\left[\begin{array}{llll}
\Delta A(k) & \Delta A_{1}(k) & \Delta B_{1}(k) & \Delta B_{2}(k)
\end{array}\right]} \\
& =D F(k)\left[\begin{array}{llll}
E_{a} & E_{a 1} & E_{b 1} & E_{b 2}
\end{array}\right],
\end{aligned}
$$

where $D, E_{a}, E_{a 1}, E_{b 1}$ and $E_{b 2}$ are known real constant matrices and $F(k) \in \Re^{p \times p}$ is an unknown matrix satisfying $F^{T}(k) F(k) \leq I_{p}$. On the other hand, $K(k) \in \Re^{q \times q}$ is the 


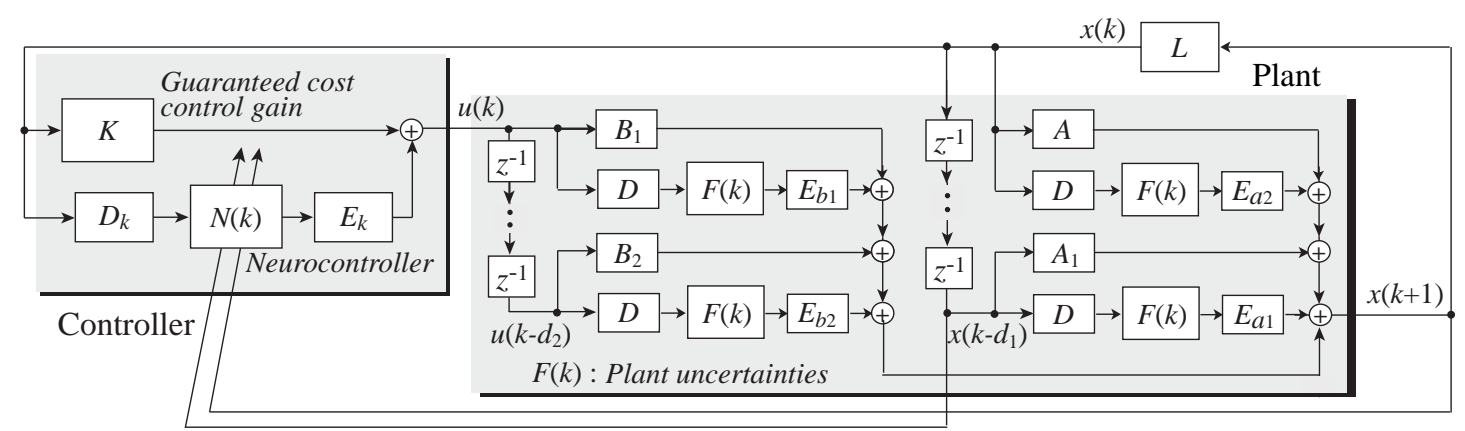

Fig. 1. Block diagram of proposed system.

state feedback gain matrix with additive gain perturbation and can be described as follows

$$
K(k)=K+\Delta K(k),
$$

where $K$ is the fixed state feedback gain and $\Delta K(k)$ represents the additive gain perturbation of the form

$$
\Delta K(k)=D_{k} N(k) E_{k} .
$$

Here $D_{k}$ and $E_{k}$ are known real constant matrices and $N(k)$ is the output of NN satisfying $N^{T}(k) N(k) \leq I_{q}$.

Associated with the system (1) is the cost function

$$
J=\sum_{k=0}^{\infty}\left[x^{T}(k) Q x(k)+u^{T}(k) R u(k)\right],
$$

where $Q$ and $R$ are given as positive definite symmetric matrices. In this situation, the definition of the guaranteed cost control with the additive gain perturbations is given below (see e.g. [1]).

Definition 1: For the uncertain system (1) and cost function (4), suppose that there exist a control gain matrix $K$ and a positive scalar $J^{*}$ such that the closed-loop systems is asymptotically stable and the closed-loop value of the cost function (4) satisfies $J<J^{*}$. If such condition is met, $J^{*}$ is said to be a guaranteed cost and $K$ is a guaranteed cost control gain matrix of the uncertain systems (1) and cost function (4).

\section{LMI-BASED DESIGN APPROACH FOR GUARANTEED COST CONTROLLER}

The objective of this section is to design the fixed guaranteed cost control gain matrix $K$ for the uncertain system (1) by means of the LMI design approach. The following theorem gives the sufficient condition for existence of the guaranteed cost control.

Theorem 1: Consider the uncertain time-delay systems (1) and the cost function (4). For the admissible uncertainties and the gain perturbations, if the LMI (5) has the feasible solutions such as the symmetric positive definite matrix $X \in \Re^{n \times n}$ and $Y \in \Re^{m \times n}$, and positive scalar $\varepsilon_{i}>0, i=1,2$, then $K=Y X^{-1}$ is the guaranteed cost control gain matrix.

Furthermore, the corresponding value of the cost function (4) satisfies the following inequality (6) for all admissible uncertainties and the gain perturbations

$$
\begin{aligned}
J<J^{*}= & x^{T}(0) X^{-1} x(0)+\sum_{i=1}^{d_{1}} x^{T}(-i) L^{-1} x(-i) \\
& +\sum_{j=1}^{d_{2}} u^{T}(-j) M^{-1} u(-j) .
\end{aligned}
$$

Proof: Applying the state feedback controller (2) to the system (1), the following closed-loop system can be derived.

$$
\begin{aligned}
x(k+1)= & {\left[A(k)+B_{1}(k) K(k)\right] x(k)+A_{1}(k) x\left(k-d_{1}\right) } \\
& +B_{2}(k) K(k) x\left(k-d_{2}\right) .
\end{aligned}
$$

Let us define the following Lyapunov function candidate

$$
\begin{aligned}
V(x(k))= & x^{T}(k) P x(k)+\sum_{i=1}^{d_{1}} x^{T}(k-i) S x(k-i) \\
& +\sum_{j=1}^{d_{2}} u^{T}(k-j) T u(k-j),
\end{aligned}
$$

where $P, S$ and $T$ are symmetric positive definite matrices. Then, the corresponding difference along any trajectory of the closed-loop system (7) is defined by

$$
\begin{aligned}
\Delta V(k) & =V(k+1)-V(k) \\
& <-x^{T}(k)\left(Q+K^{T}(k) T K(k)\right) x(k) .
\end{aligned}
$$

Thus, the matrix inequality (10) can be derived. Let us introduce the matrices $X=P^{-1}, L=S^{-1}$ and $M=T^{-1}$. The rest of the proof can be done by using the similar technique in [7], it is omitted.

Note the upper bound in (6) depends on the initial condition of the system (1). In this paper, to remove this dependence, the deterministic method [1] is adopted.

Theorem 2: If the LMI (5) has feasible solutions $X, Y, M$, $N, \varepsilon_{1}$ and $\varepsilon_{2}$, then the state feedback control law

$$
u(k)=Y X^{-1} x(k)
$$

is the guaranteed cost control law and the corresponding closed-loop cost function satisfies

$$
J \leq\left(1+d_{2}^{*}\right) \lambda_{\max }\left(U^{T} X^{-1} U\right)+d_{1}^{*} \lambda_{\max }\left(U^{T} L^{-1} U\right),
$$

where $\lambda_{\max }(\cdot)$ denotes the maximum eigenvalue of matrix, $U$ is a given matrix. 
$\Omega=\left[\begin{array}{c}\Sigma \\ \left(A X+B_{1} Y\right)^{T} \\ L A_{1}^{T} \\ M B_{2}^{T} \\ \varepsilon_{2} E_{b 1} D_{k} D_{k}^{T} B_{1}^{T} \\ 0 \\ \varepsilon_{2} D_{k} D_{k}^{T} B_{1}^{T} \\ 0 \\ \varepsilon_{2} D_{k} D_{k}^{T} B_{1}^{T} \\ 0 \\ 0 \\ 0, \quad 0\end{array}\right.$

$\begin{array}{cccc}A X+B_{1} Y & A_{1} M & B_{2} N & \varepsilon_{2} B_{1} D_{k} D_{k}^{T} E_{b 1}^{T} \\ -X & 0 & 0 & \left(E_{a} X+E_{b 1} Y\right)^{T} \\ 0 & -L & 0 & L E_{a 1}^{T} \\ 0 & 0 & -M & M E_{b 2}^{T} \\ E_{a} X+E_{b 1} Y & E_{a 1} L & E_{b 2} M & \Theta \\ X & 0 & 0 & 0 \\ Y & 0 & 0 & \varepsilon_{2} D_{k} D_{k}^{T} E_{b 1}^{T} \\ X & 0 & 0 & 0 \\ Y & 0 & 0 & \varepsilon_{2} D_{k} D_{k}^{T} E_{b 1}^{T} \\ E_{k} X & 0 & 0 & 0 \\ 0 & 0 & 0 & 0\end{array}$

$\begin{array}{cc}0 & \varepsilon_{2} B_{1} D_{k} D_{k}^{T} \\ X & Y^{T} \\ 0 & 0 \\ 0 & 0 \\ 0 & \varepsilon_{2} E_{b 1} D_{k} D_{k}^{T} \\ -L & 0 \\ 0 & -M \\ 0 & 0 \\ 0 & \varepsilon_{2} D_{k} D_{k}^{T} \\ 0 & 0 \\ 0 & \varepsilon_{2} D_{k}^{T}\end{array}$

0
$X$
0
0
0
0
0
$-Q^{-1}$
0
0
0
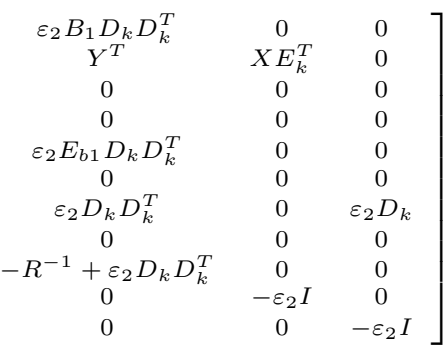

where $\Sigma=-X+\varepsilon_{1} D D^{T}+\varepsilon_{2} B_{1} D_{k} D_{k}^{T} B_{1}^{T}, \Theta=-\varepsilon_{1} I+\varepsilon_{2} E_{b 1} D_{k} D_{k}^{T} E_{b 1}$

$$
\left[\begin{array}{c}
x(k) \\
x\left(k-d_{1}\right) \\
u\left(k-d_{2}\right)
\end{array}\right]^{T}\left[\begin{array}{ccc}
\Pi & {\left[A(k)+B_{1}(k) K(k)\right]^{T} P A_{1}(k)} & {\left[A(k)+B_{1}(k) K(k)\right]^{T} P B_{2}(k)} \\
A_{1}^{T}(k) P\left[A(k)+B_{1}(k) K(k)\right] & A_{1}^{T}(k) P A_{1}(k)-S & A_{1}^{T}(k) P B_{2}(k) \\
B_{2}^{T}(k) P\left[A(k)+B_{1}(k) K(k)\right] & B_{2}^{T}(k) P A_{1}(k) & B_{2}^{T}(k) P B_{2}(k)-T
\end{array}\right]\left[\begin{array}{c}
x(k) \\
x\left(k-d_{1}\right) \\
u\left(k-d_{2}\right)
\end{array}\right]
$$

$<0$

$$
\text { where } \Pi=\left[A(k)+B_{1}(k) K(k)\right]^{T} P\left[A(k)+B_{1}(k) K(k)\right]+S+K^{T}(k) T K(k)+Q+K^{T}(k) R K(k)
$$

Proof: Since the proof can be done by using the similar technique in [2], it is omitted.

Various efficient convex optimization algorithms can be applied because the LMI (5) consists of a convex solution set. Moreover, its solutions represent the set of the guaranteed cost control gain matrix $K$. Consequently, let us consider the optimization problem that allows us to determine the optimal bound.

Theorem 3: Consider the LMI (5) and the following constrained conditions.

$$
\left[\begin{array}{cc}
-\alpha I & U^{T} \\
U & -X
\end{array}\right]<0, \quad\left[\begin{array}{cc}
-\beta I & U^{T} \\
U & -L
\end{array}\right]<0
$$

The design problem of the optimal guaranteed cost controller can be formulated as the following optimization problem.

$$
\min z=\left(1+d_{2}^{*}\right) \alpha+d_{1}^{*} \beta .
$$

Proof: Since the proof can be done by using the similar technique in [2], it is omitted.

In this paper, the LMI condition (5) and (13) will be used for the optimization problem.

\section{NEURAL NETWORKS FOR ADDITIVE GAIN PERTURBATIONS}

The LMI approach for the uncertain time-delay systems usually results in a conservative controller design due to the existence of the uncertainties and gain perturbations. The main purpose of this paper is to introduce $\mathrm{NN}$ as additive gain perturbations into the uncertain systems to improve the cost performance. Note that the proposed neurocontroller regulates its outputs in real-time under the robust stability guaranteed by the LMI approach.

It can be much expected that the reduction of the cost will be attained when the neurocontroller succeeds in managing the uncertain systems as the nominal linear systems. Therefore, let us consider the following nominal system.

$$
\begin{aligned}
\hat{x}(k+1)= & A \hat{x}(k)+A_{1} \hat{x}\left(k-d_{1}\right)+B_{1} \hat{u}(k) \\
& +B_{2} \hat{u}\left(k-d_{2}\right), \\
\hat{u}^{*}(k)= & \hat{K} \hat{x}(k),
\end{aligned}
$$

where $\hat{x}(k) \in \Re^{n}$ is the state and $\hat{u}(k) \in \Re^{m}$ is the control input. $\hat{K}$ is the state feedback gain derived by the LMI approach for the nominal system (15). Comparing with the uncertain system, it is well-known that the cost performance of the nominal system is smaller. In the proposed method, the $\mathrm{NN}$ should be trained in real-time so that the state discrepancy $\|\hat{x}(k+1)-x(k+1)\|$ between the behavior of the nominal system and the uncertain time-delay systems becomes as small as possible at each step $k$. Since the proposed uncertain systems have time-delay, it is expected that more reduction of the cost would be attained when the NNs use not only the present state value but also the past state value in the uncertain systems. $N(k)$ as the equation (3) can be expressed as the nonlinear function of the state $x(k)$, the weight coefficient of NN $w(k)$ and the threshold $\theta(k)$ as follows.

$$
N(k)=f\left(x(k), x\left(k-d_{1}\right), w(k), \theta(k)\right) .
$$

An energy function $E(k)$ is defined as the discrepancy between the behavior of the nominal system and the one of the uncertain time-delay systems at step $k$. At each step, the weight coefficients are modified so as to minimize $E(k)$ given as

$$
E(k)=\frac{1}{2}(\hat{x}(k+1)-x(k+1))^{T}(\hat{x}(k+1)-x(k+1)),
$$

$E(k)$ can be calculated by using the observed state value, $x(k+1)$. If $E(k)$ can be minimized as small as possible, the discrepancy $\|\hat{x}(k+1)-x(k+1)\|^{2}$ would also be minimized so that the cost of the uncertain time-delay systems are close to the cost of the nominal system. 


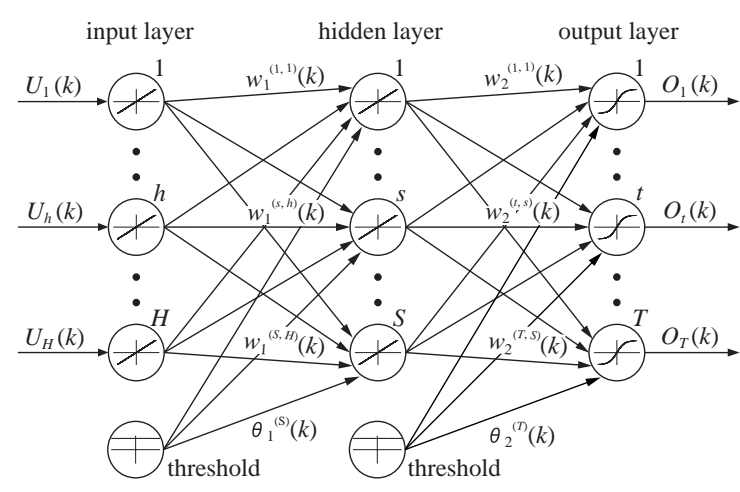

Fig. 2. Block diagram of proposed system.

In the learning of $\mathrm{NN}$, the modification of weight coefficient $\Delta w(k)$ is given by

$$
\begin{aligned}
w(k+1) & =w(k)-\eta \frac{\partial E(k)}{\partial w(k)}, \\
\frac{\partial E(k)}{\partial w(k)} & =\frac{\partial E(k)}{\partial N(k)} \frac{\partial N(k)}{\partial w(k)},
\end{aligned}
$$

where $\eta$ is the learning ratio. The term $\frac{\partial E(k)}{\partial N(k)}$ can be calculated from the energy function (18) as follows:

$$
\frac{\partial E(k)}{\partial N(k)}=-(\hat{x}(k+1)-x(k+1)) B_{1}(k) D_{k} E_{k} x(k)
$$

and $\frac{\partial N(k)}{\partial w(k)}$ depends on the NN used and can be derived using the chain rule. With (15)-(20), NN can be trained so as to decrease the cost $J$ on-line.

The utilized NN is a three-layer feed-forward network as shown in Fig. 2. A linear function is utilized in the neurons of the input and the hidden layers, and a sigmoid function in the output layer. The descriptions of each layer's input and output are the same as those in [7]. From equation (17), the input layer of $\mathrm{NN}$ is described as follows

$$
U_{N}(k)=\left[\begin{array}{ll}
x(k) & x\left(k-d_{1}\right)
\end{array}\right] .
$$

\section{NUMERICAL EXAMPLE}

In this section, the effectiveness of the proposed method is verified for the uncertain time-delay systems given below.

$$
\begin{aligned}
& A=\left[\begin{array}{ll}
2 & 1 \\
0 & 1
\end{array}\right], \quad A_{1}=\left[\begin{array}{cc}
0.2 & 0.1 \\
0 & 0.1
\end{array}\right], \quad B_{1}=\left[\begin{array}{l}
1 \\
1
\end{array}\right], \\
& B_{2}=\left[\begin{array}{l}
0.1 \\
0.1
\end{array}\right], \quad D=\left[\begin{array}{l}
2 \\
2
\end{array}\right], \quad E_{a}=\left[\begin{array}{ll}
0.2 & 0.3
\end{array}\right], \\
& E_{a 1}=0, \quad E_{b 1}=0.4, \quad E_{b 2}=0.2, \quad F(k)=1.0, \\
& D_{k}=\left[\begin{array}{cc}
0.3 & 0.2
\end{array}\right], \quad E_{k}=0.4 \\
& N(k)=\left[\begin{array}{cc}
N_{1}(k) & 0 \\
0 & N_{2}(k)
\end{array}\right], \quad d_{1}^{*}=2, \quad d_{2}^{*}=5, \\
& U=\operatorname{diag}(1.51 .5) .
\end{aligned}
$$

The initial condition is $x(0)=\left[\begin{array}{ll}2 & -2\end{array}\right]^{T}$, and the weighting matrices are chosen as $Q=\operatorname{diag}(1,1)$ and $R=1.0$, respectively.
The state feedback control gain $K$ which is based on the proposed LMI design method with the neurocontroller is given by

$$
K=\left[\begin{array}{ll}
K_{1} & K_{2}
\end{array}\right]=\left[\begin{array}{ll}
-1.3915 & -0.9722
\end{array}\right] .
$$

For the system without uncertainties (15), the state feedback control gain $\hat{K}$ is obtained as follows:

$$
\hat{K}=\left[\begin{array}{ll}
\hat{K}_{1} & \hat{K}_{2}
\end{array}\right]=[-1.4522-1.0000] .
$$

For the system without the proposed neurocontroller, that is, $N(k) \equiv 0$, the state feedback control gain $\bar{K}$ which is based on the existing method [3] is designed.

$$
\bar{K}=\left[\begin{array}{ll}
\bar{K}_{1} & \bar{K}_{2}
\end{array}\right]=\left[\begin{array}{ll}
-1.3898 & -0.9908
\end{array}\right] .
$$

The neurocontroller is composed of 30 neurons in the hidden layer, four neurons in the input layer, and two neurons in the output layer, respectively. The learning ratio is set as $\eta=0.8$. The initial weights are randomly set in the range of $[-0.05,0.05]$.

The cost $J$ of the proposed method is 115.4286 , while the cost $\bar{J}$ without the neurocontroller is 129.5551 . The cost $\hat{J}$ of the system without uncertainties is 107.2273 . It is easy to find that the proposed method make the cost of the uncertain time-delay systems reduce by using neurocontroller.

\section{CONCLUSION}

In this paper, the application of NNs for the discrete-time uncertain systems that have in both state and input delays have been investigated. To reduce the cost, the NNs has been substituted for the gain perturbations. Moreover, using the LMI approach, the robust stability of closed-loop delay systems is guaranteed even if the systems include NN. Numerical examples have shown that the proposed method is effective for the uncertain time-delay systems.

\section{REFERENCES}

[1] I. R. Petersen, D. C. McFarlane, and M.A. Rotea, "Optimal guaranteed cost control of discrete-time uncertain linear systems," Int. J. Robust Nonlinear Control, vol.8, iss.8, pp. 649-657, 1998.

[2] L. Yu, and F. Gao, "Optimal guaranteed cost control of discrete-time uncertain systems with both state and input delays," J. Franklin Institute, vol.338, iss.1, pp.101-110, 2001.

[3] W.- H. Chen, Z.- H. Guan and X. Lu, "Delay-dependent guaranteed cost control for uncertain discrete-time systems with both state and input delays", J. Franklin Institute, vol.341, iss.5, pp.419-430, 2004.

[4] G.-H. Yang, J. L. Wang and Y. C. Soh, "Guaranteed cost control for discrete-time linear systems under controller gain perturbations", Linear Algebra and its Applications, vol.312, iss.1-3, pp.161-180, 2000.

[5] K. S. Narendra and K. Parthasarathy, "Identification and control of dynamical systems using neural networks", IEEE Trans. Neural Networks, vol.1, no.1, pp.4-27, 1990.

[6] Y. Iiguni, H. Sakai and H. Tokumaru, "A nonlinear regulator design in the presence of system uncertainties using multilayered neural networks", IEEE Trans. Neural Networks, vol.2, no.4, pp.410-417, 1991

[7] H. Mukaidani, Y. Ishii, Y. Tanaka, N. Bu and T. Tsuji, "LMI based neurocontroller for guaranteed cost control of discrete-time uncertain system," Proc. 43th IEEE Conf. Decision and Control, Bahamas, Dec. 2004 (to appear).

[8] Y. Ishii, H. Mukaidani, Y. Tanaka, N. Bu and T. Tsuji, "LMI based neurocontroller for output-feedback guaranteed cost control of discretetime uncertain system," Proc. IEEE Int. Midwest Symp. Circuits and Systems, vol. III, pp.141-144, Hiroshima, July 2004. 